psychopharmacotherapy). Previous research on the effectiveness of formal depression care suggests that treatments should be more individually tailored. Tailoring treatments, however, is a time-consuming process which may hinder the implementation in LTC. A more integrative approach targeting specific groups of nursing home residents is therefore preferred and may benefit both residents and staff. To do so, insight in moderator effects is needed to better understand and better match treatments to specific groups of LTC residents.

Objectives: The aim of this study is to provide insight into (1) the effectiveness of interventions, (2) the influence of residents' characteristics (e.g., residents with cognitive impairment versus residents with physical disabilities) and/or (3) the influence of contextual factors (e.g., group-based versus individual therapy) which may have an impact on the effectiveness of interventions.

Eligibility criteria: Various databases (e.g., EBSCO, PubMed, COHRANE Library) are searched using a predefined search string, combining terms concerning our PICO elements (e.g., "Nursing Home Residents" (P), "Treatment" (I), "Depression" (O)). We only include a) randomized controlled trials investigating the use of formal depression treatments (independent variable), b) among LTC residents and, c) having used a standardized measurement tool for, d) depression (dependent variable). Results of this systematic search will be presented.

Method of synthesis: Eligible studies will further be screened and assessed for residents' characteristics and/or contextual factors. A random-effects model will be used to calculate the pooled standardized mean difference (SMD) and to assess the strength of the effects of formal depression treatments on depression. Further subgroup-analysis and meta-regressions will be used to assess the potential moderator effects.

Conclusion: To better match treatments to LTC residents, more insight into the effectiveness, moderator effects and core components of the applied interventions is needed.

\title{
517 - Informal and Formal Depression Care in Nursing Homes (InFormeD): Study protocol of a six month cohort study to better match treatment with residents
}

Abstract

Author List: Ine Declercq, Ruslan Leontjevas, Inge Knippenberg, Susan Van Hooren, Patricia De Vriendt, Debby Gerritsen

Background: Depression is common among nursing home residents and has a considerable impact on their quality of life. Therefore, there has been an increased interest in interventions aiming at the reduction of depression among nursing home residents. These interventions could be categorized into formal and informal depression care. Formal care includes psychosocial, psychotherapeutic and/or (neuro-)biological interventions. Informal care can be provided by nursing home staff, alongside the formal care (e.g., letting sunlight into the room when one believes in the positive effects of daylight). Although many studies have been done about depression treatment in nursing homes, there is still a lack of insight into the effectiveness of interventions and how they differ among specific target groups (e.g., residents with cognitive impairment versus residents with physical disabilities). Moreover, research into informal care is rather rare. More insight is needed into the effect of formal and informal depression care and the mutually reinforcing effects of those strategies on nursing home residents. This insight is essential to better match treatments with residents and to provide a more comprehensive approach to counter depression.

Objectives: The aim of this study is to gain insight in the use of formal and informal depression care and their associations with depression among nursing home residents. 
Design: A six month cohort study will be conducted.

Method: Residents will be recruited in nursing homes across the Netherlands and Flanders (Belgium). To measure formal and informal care, newly developed tools will be cross-culturally validated: one to assess the provided formal care in nursing homes, two tools for measuring the used informal strategies. Depression outcomes will be measured with the Geriatric Depression Scale, Cornell Scale for Depression in Dementia, and the Nijmegen-Observer-Rated Depression-scale). Baseline measurements and crosssectional analyses will be performed and repeated after six months. The intended associations will be assessed using multiple regression analysis.

Conclusion: To develop a good depression care policy, a more comprehensive approach is needed and may benefit both residents and staff.

\section{8 - AUTOIMMUNE DEMENTIA - WHEN TO SUSPECT?}

\section{Author list: Filipa Caetano, Margarida Araújo, Ana Samouco, Serafim Carvalho}

Background: Autoimune dementias are underrecognized clinical entities, frequently misdiagnosed as neurodegenerative or prion disorders. However, the prognosis is vastly different since immunotherapy can treat these conditions and restore functionality.

Research objective: To reflect on autoimmune dementias, briefly presenting the autoimmune syndromes, how to diagnose them and some clinical cues to be attentive of.

Methods: Literature search on Pubmed and Google Scholar.

Results: The incidence and prevalence of autoimmune dementias are unknown, but autoimmune and inflammatory causes account for $20 \%$ of dementia in patients younger than 45 years of age.

Autoimmune dementias are classified according to syndromic presentation, specific serologic markers, or histopathologic findings.

Patients with autoimmune dementias usually present with an acute or subacute disorder of memory, thinking, or behaviour. Clinical clues that can help clinicians identify autoimmune dementias include six of the following: (i) rapidly progressive or fluctuating course; (ii) multifocal and diverse clinical presentations; (iii) personal or family history of autoimmunity; (iv) detection of inflammatory markers in the cerebrospinal fluid; (v) presence of a neural-specific autoantibody and (vi) favourable response to a trial of immunotherapy. Also, unsuspected cancer, new or recurrent, may manifest neurologically as autoimmune dementia.

In evaluating patients with dementia and autoimmune disease, clinicians should be aware of the possible coexistence of these disorders.

Conclusions: Recognition of clinical and serologic clues to autoimmune dementia allows early and sustained treatment. Misdiagnosis of a potentially reversible condition as a progressive neurodegenerative disorder can have devastating consequences for the patient and family. 